Means, Gardiner C. (1962): Pricing Power and the Public Interest, New York: Harper

Mirowski, Phillip E. (1989): More Heat than Light. Economics as Social Physics, Physics as Nature's Economics, Cambridge: Cambridge University Press

Reder, Melvin W. (1999): Economics. The Culture of a Controversial Science, Chicago: University of Chicago Press

Rothschild, Kurt W. (200I): A Note on Economic Imperialism, in: Jahrbücher für Nationalökonomie und Statistik, Vol. 22I, No. 4, pp. 440-447

Selten, Reinhard (1990): Bounded Rationality, in: Journal of Institutional and Theoretical Economics, Vol. i46, No. 4, pp. 694-658

Sen, Amartya K.(1987): Rational Behaviour, in: The New Palgrave Dictionary of Economics, London: Macmillan, Vol. IV, pp. 68-76

Simon, Herbert A. (1957): Models of Man. Social and Rational, New York: Wiley

\title{
Post-Autistic Economics. Eine studentische Intervention für plurale Ökonomik Thomas Dürmeier*
}

Arbeitslosigkeit, Wettbewerbsfähigkeit oder Wirtschaftswachstum bestimmen die Presseschlagzeilen. Bei der Erklärung dieser gesellschaftlichen Herausforderungen greifen Ökonom|inn|en auf abstrakte Begrifflichkeiten und mathematische Modelle zurück, die überwiegend dem neoklassischen Paradigma entspringen. Auch Studierende der Wirtschaftswissenschaften verlieren das Vertrauen, mit dieser herrschenden Theorieschule überzeugende Antworten zu finden und die gesellschaftliche Realität zu entschlüsseln (vgl. Colander/ Klamer 1987). Seit dem Jahr 2000 engagieren sich Studierende, Intellektuelle und Professor|inn|en in der Protestbewegung "Autisme-Économicie«, um gegen den wissenschaftlichen Autismus der Mainstream-Ökonomik zu intervenieren und eine »Post-Autistische Ökonomik" einzufordern.

\section{Die Geschichte der Post-Autistischen Studierendenbewegung}

Wissenschaftliche Innovationen entstehen normalerweise dann, wenn Professor|inn|en neue Paradigmen an den Hochschulen in einer "scientific revolution" (Kuhn I962) durchsetzen. Die Post-Autistische Ökonomik hingegen erblickte das Licht der Welt, als Studierende mit ihrem Studienalltag nicht mehr zurechtkamen.

* Universität Kassel. Besonderer Dank gilt Tanja von Egan-Krieger und Helge Peukert für die freundliche Unterstützung. 
Alles begann sehr unspektakulär im Juni 2000 an der Sorbonne in Paris. In einer Petition »Autisme-Économie» (Autisme-Économicie 2000) protestierte eine kleine Gruppe von Wirtschaftsstudierenden gegen "autistische Wissenschaft« im Internet. Die unkontrollierte Anwendung der Mathematik und formaler Modelle dürften nicht Selbstzweck sein. Sie forderten Wissenschaft statt Szientismus, Pluralismus statt neoklassischem Monotheismus, empirischen Realismus statt deduktiver Abstraktionen und riefen ihre Professor|inn|en auf, die Ökonomik aus ihrem autistischen und sozial unverantwortlichen Zustand zu retten (vgl. Fullbrook 200I). Die Protestierenden forderten eine "Économie Post-Autiste« (AutismeÉconomicie 200I).

Eine kleine Gruppe von Lehrenden unterstützte diese Kritik. Der Abdruck der studentischen Petition in der Tageszeitung Le Monde trat eine landesweite Debatte los. Innerhalb von zwei Wochen hatten sich 600 Studierende mit ihrer Unterschrift hinter ihre Kolleg|inn|en gestellt. Eine Radiosendung am 2r. September 2000 brachte dann den wissenschaftspolitischen Durchbruch. Der französische Minister für Erziehungsfragen, Jack Lang, reagierte und setzte eine Kommission zur Überarbeitung der landesweit einheitlichen Curricula ein. Parallel hierzu gründete Edward Fullbrook den elektronischen »Post-Autistic Economics Newsletter" (später Post-Autistic Economics Review), der sich innerhalb kürzester Zeit zu einem wissenschaftlichen Journal weiterentwickelte.

Der orthodoxe Mainstream konnte diese Reformbewegung nun nicht länger ignorieren. Am 3I. Oktober meldeten sich der Makroökonom Olivier Blanchard, der Nobelpreisträger Robert Solow und fünfzehn Ökonom|inn|en, wie Christian de Boissieu oder Jean-Jacques Rosa, zu Wort und lehnten öffentlich in Le Monde in ihrer Gegenpetition "Contre-appel pour préserver la scientificité de l'économie« die post-autistischen Ideen ab, ${ }^{\mathrm{I}}$ wobei sie auf die Argumente der Studierenden nicht eingingen. Fullbrook bewertete diese Stellungnahme als zynische Fehlinterpretation der studentischen Anliegen.

"Most of all, however, people on all sides seemed surprised at how feeble were the arguments offered for blocking the reforms proposed by the PAE Movement." (Fullbrook 200I)

James Kenneth Galbraith kam nach Paris und unterstützte die Studierenden. Die Gegenpetition stelle auch den besten Beweis dar, dass die französischen Studierenden mit ihrem Appell für eine fundamentale Reform im Recht sind (vgl. Galbraith 200I).

Die französische Studierendenbewegung internationalisierte sich nun immer stärker. Im November 2000 starteten auch Politikwissenschaftler|innen in den Vereinigten Staaten das »Perestroika-Movement « gegen das Übergewicht von Public-Choice-Ansätzen. Im Winter 200I gründeten sich Gruppen für Post-Autistische Ökonomik in Australien und China, in der Türkei und Spanien. Im Juni desselben Jahres unterzeichneten 27 Doktorand|inn|en der Cambridge University die Petition »Open up economics.»

I Die Gegenposition ist im Internet abrufbar unter: http://mouv.eco.free.fr/mouvement/tcontre. htm. 
"We are not arguing against the mainstream approach per se, but against the fact its dominance is taken for granted in the profession. We are not arguing against mainstream methods, but believe in a pluralism of methods and approaches justified by debate." (The Cambridge 27: 200I)

Auf zahlreichen Tagungen diskutierten Ökonom|inn|en über die Ideen der Post-Autist|in$\mathrm{n} \mid$ en. Zahlreiche heterodoxe Konferenzen, wie beispielsweise in Kansas City, solidarisierten sich mit der Post-Autistik.

Der Abschlussbericht »L'Enseignement supérior de l'économie en question« der französischen Kommission stimmte ihnen zu, indem er die Integration einer Diskussion über gegenwärtige Wirtschaftsthemen in die Struktur und Inhalte universitärer Veranstaltungen in der Volkswirtschaftslehre forderte (vgl. Fullbrook 20oI). Der Bericht erweckte allerdings nicht den Anschein, der Beendigung der Hegemonie der neoklassischen Mikroökonomik zuzustimmen (vgl. Benicourt 200I). Selbst diese ersten kleinen Reformansätze wurden jedoch nach dem Regierungswechsel nicht umgesetzt.

Die weltweite Dynamik ging aber weiter voran und spiegelt sich im Post-Autistic Economics Review wider, das bis heute Zuspruch von 8.I2I Abonnent|inn|en aus über I5o Ländern fand. Zu den Autor|inn|en zählten u.a. Herman Daly, Deirdre McCloskey, Tony Lawson, Geoff Harcourt oder Julie A. Nelson. Diese Erfolgsgeschichte stellt eine der größten Herausforderungen für die Orthodoxie seit der Keynesianischen »Revolution« dar. „Economics has not experienced such pressure to change since the I930s." (Fullbrook 200I)

\section{Initiativen des bundesdeutschen Arbeitskreises Post-Autistische Ökonomie}

Deutschland verschlief lange die französische Revolte und die internationale Dynamik der Post-Autistik. Die Post-Autistik wurde am 3. April 2002 erstmals in der Süddeutschen Zeitung als »Gestörte Wirtschaftsbeziehungen« erwähnt. Erst auf der Attac-Sommerakademie 2003 entstand eine studentische Initiative für eine Post-Autistische Ökonomik in der Bundesrepublik. Eine kleine Gruppe von Studierenden aus Berlin, Heidelberg und Regensburg gründete am I6. November 2003 in Heidelberg den Arbeitskreis Post-Autistische Ökonomie. Durch Vernetzungs- und Informationsaktivitäten entstanden eine erste wissenschaftliche Tagung und zahlreiche Workshops zu Wissenschaftstheorie, Wachstum, Postkeynesianismus, Evolutorik oder Ökologische Ökonomik. Über das verabschiedete Positionspapier und Ergebnisse berichtete bislang jedoch nur die »Tageszeitung" am 24. Mai 2004.

Ob die Post-Autistische Ökonomik eine Zukunft im deutschsprachigen Raum hat, wird sich zeigen. Zahlreiche Projekte und Ortsgruppen sind im Entstehen. Eine Vernetzung mit der studentischen Initiative »Kritische Wirtschaftswissenschaft « in Berlin ${ }^{2}$ und wissenschaftlichen Innovationen wie der Kulturellen Ökonomik oder der Postkeynesianischen Makroökonomik bestehen. Im Gegensatz zu den Ereignissen in Frankreich hat jedoch der

2 Die Kritische Wirtschaftswissenschaft veranstaltet Diskussionsrunden und öffentliche Veranstaltungen. Weitere Informationen unter: http://www.kritische-wirtschaftswissenschaften.de. 
Verein für Socialpolitik - als die größte Vereinigung von Wirtschaftswissenschaftler|inne|n in Deutschland - die Anliegen der Studierenden bisher nicht wahrgenommen.

\section{Die Kritik an der autistischen Orthodoxie}

Der Autismus der heutigen Orthodoxie steht in einer Linie mit der Geschichte des ökonomischen Denkens. Die (neo)-klassische Schule beruht auf der Annahme, dass im freien Wechselspiel der Eigeninteressen perfekte Märkte die gesellschaftliche Wohlfahrt maximieren. Die Neoklassik ging ohne wesentliche Veränderungen in mathematische Modellierungen über, die sich ebenso in der Spieltheorie oder der Neuen Institutionenökonomik wiederfinden (vgl. Abbildung I).

Abbildung I: Geschichte des ökonomischen Denkens

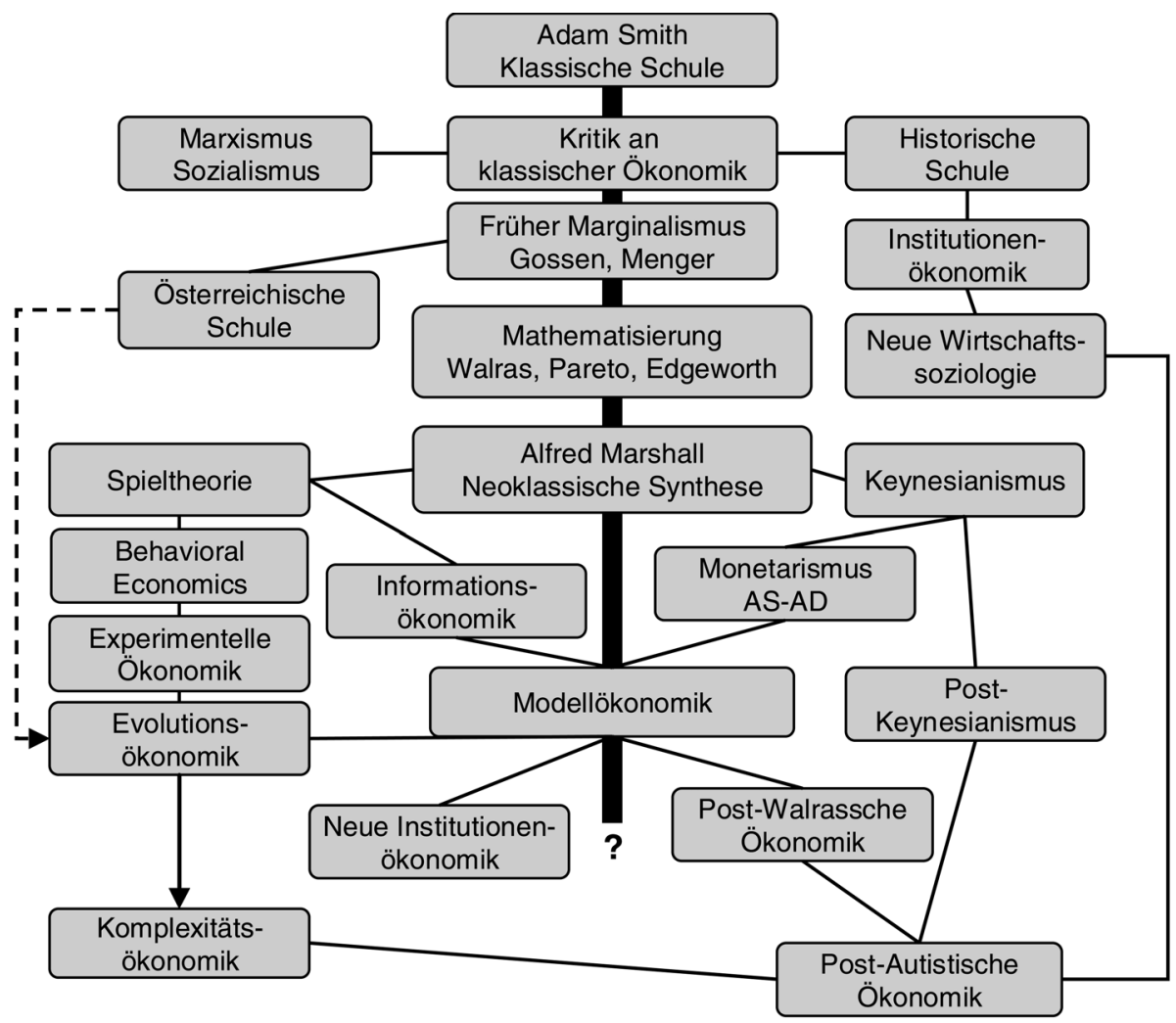

Quelle: Dürmeier 2002: 9 
Immer mehr theoretische und empirische Erkenntnisse und alternative Denkschulen widersprechen jedoch dem (neo)-klassischen Mainstream und seiner ideengeschichtlichen Kontinuität. Aktuelle Erkenntnisse der experimentellen Ökonomik oder reales Versagen der Wirtschaftspolitik wie in der Asienkrise rütteln an den Grundfesten des Mainstreams. So versucht etwa die Post-Walrassche Ökonomik (vgl. Colander [Hg.] I996, Stiglitz I993) multiple Gleichgewichte zu integrieren. Wir erleben heute eine Verteidigung des orthodoxen Paradigmas mit partiellen Modellerweiterungen gegen die empirischen Falsifikationen. ${ }^{3}$ Nach Thomas Kuhn könnte die Fragmentierung der Orthodoxie als Krise des neoklassischen Paradigmas interpretiert werden (vgl. Dürmeier 2002).

Die dennoch nach wie vor vorherrschende Stellung der orthodoxen Ökonomik lässt sich auf mehrere Ursachen zurückführen. Verbale Sozialwissenschaften können sich nur schwer gegen mathematische Eleganz durchsetzen. Mit der Entstehung des modernen Denkens in der Aufklärung lösten Mathematik und Physik Theologie und Philosophie als zentrale Wissenschaften ab. Mathematisches und mechanisches Denken wurde zum wissenschaftlichen Ideal. Die orthodoxe Wirtschaftstheorie entwickelt aus moderner Rationalität und Nützlichkeit vereinfachte Modelle und leitet daraus klare Aussagen für zahlreiche Phänomene ab, was das Vordringen des "Ökonomischen Imperialismus (vgl. Aretz 1997, Becker I976, Becker I98I) in andere Bereiche wie Politik oder Familie zeigt. Ökonomische Modelle erlauben im Gegensatz zur Soziologie schnell Prognosen und eignen sich daher besser zur Politikberatung (vgl. Smelser/Swedberg I994: 4). Die Modellvereinfachungen müssen zwar mit einem Realitätsverlust in den Modellwelten erkauft werden, die marktbeherrschende Stellung der Neoklassik in Bezug auf Publikationsmöglichkeiten, in Instituten oder hinsichtlich der finanziellen Förderung gibt ihr jedoch einen absoluten Wettbewerbsvorteil gegenüber heterodoxen Theorien.

Es stellt sich nun aber die Frage, warum sich die Orthodoxie in eine autistische Wissenschaft verwandelt hat. In der post-autistischen Literatur und der Geschichte des ökonomischen Denkens lassen sich zahlreiche und widersprüchliche Erklärungen finden. Die allgemeine Überzeugung, dass die Neoklassik die bessere Erklärung für wirtschaftliche Prozesse bietet, stimmt teilweise. Häufig kann eine neoklassische Analyse (partielle) Antworten geben. Es könnte somit sogar von einem kumulativen Wissensfortschritt gesprochen werden. Dennoch hat sich eine autistische Selbstreferentialität herausgebildet. Die Neoklassik könnte nach Kuhn das Ende ihres Paradigmas erreicht haben, und ihre Vertreter|innen versuchen nun, sich gegenüber allen Widersprüchen, Falsifikationen oder empirischen Anomalien zu immunisieren.

Andere Wissenschaften haben den Autismus »befördert«. Die Entwicklung des Computers erleichterte das neoklassische Modelldenken (vgl. Mirowski 2002). Disziplinen wie die Soziologie haben sich lange aus dem wissenschaftlichen Streit mit den Wirtschaftswissenschaften zurückgezogen. Neben diesen Faktoren hat auch die Politik die Wirtschaftswissenschaft verändert.

3 Für eine Zusammenstellung von empirischen Anomalien zum rationalen Handeln vgl. Reckling 2002: 65-I25. 
Politische Entwicklungen wie der Kalte Krieg und die Nützlichkeit der Spieltheorie zur Erklärung der in dieser historischen Phase zu beobachtenden Interaktionen zwischen den beteiligten Ländern und Länderblöcken verstärkten die Bedeutung der Neoklassik (vgl. Bernstein 200I). Andere Schulen, insbesondere kritische Richtungen, gerieten immer stärker an den Rand. Die Stagflation der I970er und der in ihrem Gefolge aufkommende Monetarismus lösten den Keynesianismus ab (vgl. Blyth 2002) und schwächten heterodoxe Positionen. Ökonomisches Denken diente besonders bis zum Ende des Ost-West-Konfliktes etwa bei den neoliberal orientierten Ökonom|inn|en der Chicago School zur Absicherung der ideologischen Hegemonie der kapitalistisch geprägten Marktwirtschaften.

Auch weitere gesamtgesellschaftliche Prozesse trugen zum Autismus der Orthodoxie bei. Das moderne Denken in Rationalität und positivistischer Statistik verengte volkswirtschaftliches Fragen auf rationales Handeln und ökonometrische Methoden. Die philosophische Kritik der Postmoderne rezipierte der Mainstream außer mit der Arbeit von McCloskey (1985) kaum. Der Kritische Realismus (vgl. Lawson 2003) wird bis heute nur in der Heterodoxie berücksichtigt. Die gestiegene Komplexität und Fragmentierung der Wirtschaftswissenschaft trugen zu einer Autopoiesis, einem Selbstbezug des wissenschaftlichen Systems der Mainstreamökonomik, bei.

Aus wissenschaftssoziologischer Perspektive sind evolutionäre Prozesse, die Sprachspiele und die Machtlogik des volkswirtschaftlichen Feldes zu berücksichtigen. Insbesondere die Volkswirtschaftslehre hat eine intellektuelle Pfadabhängigkeit im wissenschaftlichen Denken ausgebildet, die zu einer Reproduktion fast ausschließlich der heutigen Lehrmeinungen führt (vgl. Marietta/Perlman 200o). Die zentrale Stellung der Lehrbücher in der Ausbildung, die Marginalisierung von Ideengeschichte und der Verzicht auf das Studieren von Originaltexten verdeutlichen dies. Mit der Feldtheorie von Pierre Bourdieu kann man sagen, dass im volkswirtschaftlichen Feld die mathematischen Naturwissenschaften einen höheren symbolischen Wert besitzen. ${ }^{4}$ Es gibt einen besonderen Habitus in der Ökonomik. Mathematische Sprache dient zur sozialen Abgrenzung. Hierarchien und gegenseitige Abhängigkeiten (vgl. Siler 2003) lassen spezifisches Sozialkapital und Netzwerke entstehen, mit denen sich der Mainstream von anderen Gruppen abgrenzt. Im Graubereich zwischen orthodoxer und heterodoxer Ökonomik entstehen jedoch oft wissenschaftliche Innovationen, wie etwa die Arbeiten von Akerlof zeigen (vgl. Foldvary 1996). Diese Grenzgänger|innen im Spannungsfeld verschiedener Schulen stehen aber einem Trade-off aus orthodoxer Akzeptanz und innovativer Kritik gegenüber (vgl. Frey/Eichenberger 1997).

Die Mathematik spielt bis heute eine besondere Rolle für den Autismus in der Mainstream-Ökonomik. Mathematische Modelle haben gegenüber verbalen Darstellungen zahlreiche Vorteile. Logische Inkonsistenzen kann man leicht erkennen, Variablen sind eindeutige und reduzierte Definitionen, und aus einem mathematischen System können leicht weitere Aussagen abgeleitet werden (vgl. Buchholz 1993, Boland 1996). Mathematik erlaubt

4 Die aktuelle Übertragung der Hirnforschung aus den naturwissenschaftlichen Teilgebieten der Psychologie als "Neuroeconomics" (vgl. Camerer et al. 2005) auf die Wirtschaftswissenschaft zeigt z.B. die Dominanz naturwissenschaftlicher Werte. 
auch, Interdependenzen zwischen Variablen sehr kompakt darzustellen. ${ }^{5}$ Verbalisierte Theorien hingegen verwenden offenere und oft mehrdeutige Begriffe, die einer Interpretation bedürfen. Sprache erlaubt damit die Darstellung von Strukturen, die sich nur schwer mathematisch darstellen lassen, und verbale Formulierungen ermöglichen aufgrund ihrer Offenheit kreative Weiterentwicklungen. Formale und verbale Darstellungen haben somit ihre je eigenen Vor- und Nachteile (vgl. Woo I986).

Widerstand gegen neoklassische Ansätze regte sich schon lange. Heute fordern auch Studierende mit dem »Kampfbegriff" post-autistische Ökonomik eine andere Forschung und Lehre. Der aus der Psychologie entlehnte Begriff »Autismus" charakterisiert eine innere Zurückgezogenheit, um sich vorwiegend in der Welt seiner eigenen Vorstellungen und Phantasie zu bewegen. Soziale Kontakte und ihre Kommunikationsfähigkeiten sind stark eingeschränkt, Interessen verzerrt und das Verhalten oft abnormal. Einzelne Begabungen wie mathematisches Denken oder Musik können extrem ausgeprägt sein. Autismus kann man in der heutigen Mainstreamökonomik sowohl im Menschenbild des homo oeconomicus als auch im Wissenschaftsalltag des neoklassischen Mainstreams feststellen.

Karl Polanyi wies bereits in seinen wirtschaftsanthropologischen Studien auf den Unterschied von formaler und substantieller Ökonomik hin, wobei letztere den Menschen in soziale Beziehungen einbettet (vgl. Polanyi I957, Dürmeier 2002). Der Mensch steht in ständigen Wechselbeziehungen mit seiner sozialen Umwelt und kann nicht als atomistisches Wesen wissenschaftlich sinnvoll gedacht werden, wie es der methodische Individualismus behauptet. Externe Effekte, Sozialkapital, Institutionen usw. müssten ständige Bestandteile des Grundmodells sein.

Die heutige Wirtschaftswissenschaft arbeitet autistisch in ihren eigenen »mondes imaginaires (Autisme-Économicie 200I), wesentliche Elemente wie die Rationaliät des homo oeconomicus werden kaum empirisch überprüft. Erst die experimentelle Ökonomik hat heute zahlreiche "empirische Anomalien" aufgedeckt. Es stellt sich auch die Frage, ob die ökonometrischen Schätzungen von Modellen, die aufgrund der begrenzten empirischen Daten stark vereinfacht werden, die Aussagekraft heutiger Modelle untermauern können. Der Mainstream berücksichtigt oft nur die neoklassische Weltsicht in theoretischer wie methodischer Hinsicht. Interdisziplinarität und Dialog mit anderen Schulen wird weitestgehend vermieden. In einer autistischen Inselbegabung strebt man nach einer mathematischen Physik der Wirtschaft. Es mutet beinahe so an, als ob mathematische Modelle die Trennung von der empirischen Realität überkompensieren sollen. ${ }^{6}$ Die neoklassische Mathematik sollte die Schwachstellen ihrer theoretischen Fundierung erkennen (vgl. Keen 200I).

Auch die heutige Lehre beinhaltet antiquierte und autistische Züge. "Scholastische» Lehrbücher und verschulte Veranstaltungen bestimmen den Alltag wirtschaftswissenschaftlicher Fakultäten. Es ist heute allgemein üblich, Ökonom|in zu werden, ohne ein einziges

5 So ist beispielsweise die verbale Modellentwicklung von Eli Heckscher in seinem Aufsatz »The Effect of Foreign Trade on the Distribution of Income« (Ekonomisk Tidskrift, 1919) umfangreicher und unverständlicher als die Lehrbuchdarstellung des Heckscher-Ohlin-Modells.

6 Über die wachsende Rolle von Mathematik in den führenden Zeitschriften vgl. McCloskey (1985). 
Mal Smith, Marx, Keynes oder Hayek gelesen zu haben. Studierende können sich kaum intellektuell mit wirtschaftspolitischen Entwicklungen auseinandersetzen. Die autistische Orthodoxie reproduziert ihren Autismus im Modelldenken des Nachwuchses. Diese wissenschaftsinternen Fragen haben aber eine höchst politische Note, da auf der Grundlage einseitiger Modelle und empirisch vager Theorien politische Entscheidungsträger|innen beraten werden.

\section{Post-Autistische Alternativen ohne neues Paradigma?}

Post-Autist|inn|en kritisieren die theoretische Enge, die Realitätsferne und die methodischen Schwächen der autistischen Orthodoxie. Sie fordern daher eine fundamentale Reform von Wissenschaft, Forschung und Lehre in der heutigen Ökonomik, die heterodoxe Ökono$\mathrm{m} \mid$ inn|en bereits seit langem einfordern.

Die Post-Autistische Ökonomik will das autistische Menschenbild des homo oeconomicus überwinden. Wie die Wirklichkeit menschlichen Lebens von Solidarität und sozialem Gruppendruck geprägt ist, so soll auch die Theorie diese sozialen Züge aufnehmen. Der homo oeconomicus soll nicht nur rational, sondern auch sozial, kommunikativ und politisch handeln: Denn die Kultur, Geschichte und Gesellschaft und die hier langfristig stattfindenden Veränderungen beeinflussen den methodologisch gedachten Individualisten, und seine Präferenzen sollten sich im Spannungsfeld sozialen Lernens wandeln.

Jede Theorie muss in der Realität geerdet sein. Reale Phänomene geben Prüfsteine für neue und bessere Theorien. Deduktive Theorieableitungen aus allgemeinen Annahmen und Axiomen können erst Aussagekraft beanspruchen, wenn sie die Realität besser erklären. Die empirische Fundierung aus Erkenntnissen der Wirtschaftsgeschichte, der experimentellen Ökonomik oder anderen Wissenschaften sollten sich in Theorien und Modellen wiederfinden.

Neben einem Wandel der Forschung fordern Post-Autist|inn|en auch eine andere Lehre. Lehrbücher und Vorlesungen vermitteln nur Überblickswissen. Eine universitäre Hochschulbildung entsteht jedoch erst durch selbständiges Arbeiten und Denken und der daraus folgenden Auseinandersetzung im Seminar. Intellektuelle Bildung erfordert den Diskurs.

Die Forderung nach methodischer Vielfalt löst oft Missverständnisse aus. Solow (200I) warf den Post-Autist|inn|en vor, nicht rechnen zu wollen. Post-Autist|inn|en lehnen aber Mathematik und formale Klarheit nicht ab, sondern wollen die Grenzen formaler Modelle aufzeigen und sich nicht in mathematischen "Traumwelten « verlieren. Mathematik muss zweckdienlich sein, was auch die Integration von mathematischer Komplexität oder Chaostheorie bedeuten kann. Daneben sollten wir verlorene Methodenschätze wiederfinden. Fallstudien, historische Vergleiche, Befragungen oder Begriffsdekonstruktion ermöglichen "neue« und ebenso richtige Perspektiven auf die Realität.

All dies könnte Ökonom|inn|en besser in die Lage versetzen, relevante und zentrale gesellschaftliche Fragen zu beantworten. Lebensqualität (vgl. Sen 200o) oder die natürlichen Lebensgrundlagen des Menschen (Ökologische Ökonomik) müssen gegenüber Fragen von Effizienz und Wachstum Vorrang bekommen. 
Alle diese Forderungen laufen schließlich in der zentralen Idee des Post-Autismus zusammen. Pluralität befreit aus der autistischen Enge der Orthodoxie (vgl. Fullbrook 2005). Die Wirtschaftslehre muss wieder Raum für die Fächer Wirtschaftsgeschichte, Geschichte des ökonomischen Denkens, Wirtschaftsethik und Wissenschaftstheorie schaffen. Nur die Reflexion über das eigene wissenschaftliche Tun ermöglicht den kreativen Fortschritt einer Disziplin. Der Mainstream vergisst den Reichtum und die Vielfalt der heterodoxen Schulen und Ideen. Der freie und offene Wettbewerb und Diskurs zwischen den sich widersprechenden Schulen schafft Innovation und hinterfragt die jeweils fremde Argumentation. Interdisziplinarität erweitert den eigenen Horizont und schafft neue Kooperationsmöglichkeiten, obwohl die Sprache des anderen oft ungewohnt ist.

Wenn die orthodoxe Wirtschaftswissenschaft nur eine Hauptströmung propagiert, muss dies keine schlechte Theorie sein, aber wenn sie die alleinige Quelle der Politikberatung darstellt, führt dies letztendlich zu einem ideologischen Tunnelblick und zu politischer Dogmatik. Pluralität der Schulen und Disziplinen in der Analyse der Wirtschaft schafft nicht nur den Boden für Wettbewerb und evolutorischen Wissensfortschritt, sondern verhindert auch die politische Einseitigkeit der heutigen Politikberatung.

Der Pluralismus der Post-Autist|inn|en (vgl. Fullbrook 2005) weist zwar den Weg, aber eine alternative und bessere Metatheorie besitzen sie noch nicht. Allerdings ergeben sich je nach Forschungsfrage im offenen Diskurs über Stärken und Schwächen der heterodoxen oder innovativ-neoklassischen Schulen Alternativen zum Mainstream. ${ }^{7}$ In der postautistischen Ökonomik treffen traditionelle Institutionenökonomen wie Warren J. Samuels auf Komplexitätsökonomen wie Paul Omerod, Femistische Ökonom|inn|en auf Neomarxist|inn|en und Postkeynesianer|innen. Ebenso findet man Vertreter|innen der Ökologischen, Evolutorischen oder Kulturellen Ökonomik, der Neuen Wirtschaftssoziologie und des Kritischen Realismus.

Es gibt zahlreiche Alternativen zum Mainstream, auch wenn keine Denkrichtung die aktuell herrschende Neoklassik alleine ablösen kann. Pragmatischer Pluralismus ist somit das post-autistische Paradigma.

\section{Ein Ausblick in die Zukunft}

Die Post-Autistische Bewegung versteht ihre wissenschaftspolitische Aufgabe darin, über heterodoxe Ökonomik und Kritik am Mainstream zu informieren, alternatives Denken und Forschung zu vernetzen und öffentlichen Druck aufzubauen, um eine Reform der Orthodoxie zu befördern.

Zahlreiche Informationen und Publikationen stehen im Internet unter www.paecon. net kostenlos zur Verfügung. Es gibt bereits eine Vielzahl englischsprachiger Publikationen. Die ersten deutschsprachigen Bücher werden 2006 erscheinen. Auf der bundesdeutschen Homepage (www.paecon.de) kann man sich über die Ergebnisse der bisherigen Tagungen in

$7 \quad$ Einen ersten Vergleich und eine Evaluation der verschiedenen Schulen findet man bei Keen (200I) und Landreth/Colander (2002). 
der Bundesrepublik informieren, wo insbesondere Studierende die Möglichkeit haben, sich mit heterodoxen Ansätzen zu beschäftigen. Neben den bundesweiten Treffen entstanden auch Regionalgruppen in Berlin, Hamburg, Heidelberg oder Göttingen. Kooperationen mit anderen Gruppen entwickeln sich, und eine bundesweite Informationskampagne, die sich an Studierenden und Lehrenden orientiert, ist in Vorbereitung.

Wie die Erfolge in Frankreich, Großbritannien oder den Vereinigten Staaten zeigen, kann eine öffentliche Debatte über das Versagen des Mainstreams zu einer Reform beitragen. Unter dem Leitmotiv »taking economics into the streets« entstanden Protestbriefe und Erklärungen in zahlreichen Ländern.

Zahlreiche Fragen beschäftigen die Post-Autistik. Obwohl die neoklassische Wirtschaftslehre kritisiert wird, besitzt sie doch einen - wenn auch umstrittenen - Stellenwert für die Zukunft. Auch an Ansätzen wie der experimentellen Ökonomik oder an unkonventionellen Denker|inne|n wie Amartya Sen scheiden sich die Geister (vgl. Benicourt 2002). In der Evolutorischen Ökonomik jedoch können zahlreiche post-autistische Potentiale stecken. Obwohl ein pragmatischer Eklektizismus eine heterodoxe Synthese bilden könnte, bietet der Kritische Realismus eine neue Erkenntnistheorie und Ontologie. Die Pluralität und Akzeptanz der verschiedensten Ansätze eint die post-autistische Bewegung, obwohl sicher nicht alle Denkrichtungen die gleichen Startbedingungen im wissenschaftlichen Wettstreit haben. Neoklassische Orthodoxie besitzt eine Vormachtsstellung gegenüber heterodoxen Ansätzen. Die wissenschaftspolitischen »Kämpfe« um das bessere Argument werden aber hoffentlich längerfristig nicht durch die wissenschaftliche und politische Vormachtstellung der neoklassischen Ökonomik, den Zugang zu Drittmitteln oder die Anzahl der Lehrstuhlinhaber|innen entschieden.

\section{Literatur}

Aretz Hans-Jürgen (1997): Ökonomischer Imperialismus? Homo oeconomicus und soziologische Theorie, in: Zeitschrift für Soziologie, Jg. 26, Nr. 2, S. 79-95

Autisme-Économicie (200I): Le Movement Autisme-Économicie: Une lettre ouverte internationale à tous les départements d'économie: une invitation à la réforme, http://mouv. eco.free.fr/presse/touverte.htm (letzter Zugriff: 5. Juli 2004)

Autisme-Économicie (2000): Le Movement Autisme-Économicie: Petition, http://mouv.eco. free.fr/mouvement/tmouvement.htm (letzter Zugriff: 26. Dezember 2002)

Becker, Gary S. (198I): A Treatise on the Family, Cambridge: Harvard University Press

Becker, Gary S. (1976): The Economic Approach to Human Behavior, Chicago: University of Chicago

Benicourt, Emmanuelle (2002): Is Amartya Sen a Post-Autistic Economist?, in: Post-Autistic Economics Review, Nr. I5, 4. September, Artikel 4

Benicourt, Emmanuelle (200I): A Year in French Economics, in: Post-Autistic Economics Newsletter, Nr. 7, Io. Juli, Artikel 6

Bernstein, Michael A. (200I): Rethinking Economics in Twentieth-Century America: A PoliticalEconomic Approach to the History of Thought, in: Post-Autistic Economics Newsletter, Nr. 8, 5. September, Artikel 5 
Blyth, Mark (2002): Great Transformations, Cambridge: Cambridge University Press

Boland, Lawrence A. (1996): Realism in Economic Model Building, in: Medema, Steven G./ Samuels, Warren J. (Hg.), Foundations of Research in Economics: How Do Economists Do Economics?, Cheltenham/Northampton: Edward Elgar, S. I8-29

Buchholz, Wolfgang (1993): Die Mathematisierung der Ökonomie - die Fragwürdigkeit einer Debatte, in: Schmid-Schönbein, Thomas et al. (Hg.), Jahrbuch für Ökonomie und Gesellschaft, Die ökonomische Wissenschaft und ihr Betrieb, Band Io, Marburg, S. II-3I

Camerer, Colin/Loewenstein, George/Prelec, Drazen (2005): Neuroeconomics, in: Journal of Economic Literature, Jg. 43, Nr. I, S. 9-64

Colander, David (Hg.) (1996): Beyond Microfoundations: Post-Walrasian Macroeconomics, Cambridge/New York/Melbourne: Cambridge University Press

Colander, David/Klamer, Arjo (1987): The Making of an Economist, in: Journal of Economic Perspectives, Jg. I, Nr. 3, S. 95-III

Dürmeier, Thomas (2002): Kritiken der Neoklassik: Neoklassik, Neue Wirtschaftssoziologie und die »Post-Autistic Economics«- Bewegung und deren Menschenbilder in globalen Strukturen, Regensburg, mimeo

Foldvary, Fred (1996): Comparative Economic Theory, in ders. (Hg), Beyond Neoclassical Economics: Heterodox Approaches to Economic Theory, Cheltenham, Brookfield: Edward Elgar, S. I-2I

Frey, Bruno S./Eichenberger, Reiner (1997): Economists: First Semester, High Flyers and UFOs; in: van Bergeijk, Peter A.G. et al. (eds.), Economic Science and Practice: the Roles of Academic Economists and Policy-Makers, Cheltenham/Lyme: Edward Elgar, S. $15-48$

Fullbrook, Edward (2005): Teaching Economics: PAE and Pluralism, in: European Association for Evolutionary Political Economy's Newsletter, Nr. 34, S. 6-9

Fullbrook, Edward (200I): A Brief History of the Post-Autistic Economics Movement, http:// www.btinternet.com/ - pae_news/history.htm (letzter Zugriff: 5. August 2002)

Galbraith, James K. (200I): A Contribution on the State of Economics in France and the World, in: Post-Autistic Economics Newsletter, Nr. 4, 29. Januar, Artikel I

Keen, Steve (200I): Debunking Economics: The Naked Emperor of the Social Sciences, Annandale: Pluto Press

Kuhn, Thomas S. (1962): The Structure of Scientific Revolutions, Chicago: University of Chicago Press

Landreth, Harry/Colander, David C. (2002): History of Economic Thought, Boston, MA. et al.: Houghton Mifflin

Lawson, Tony (2003): Reorienting Economics, Economics as a Social Theory, London: Routledge

Marietta, Morgan/ Perlman, Mark (2000): The Uses of Authority in Economics: Shared Intellectual Frameworks as the Foundation of Personal Persuasion and Schools of Economic Thought, in: American Journal of Economics and Sociology, Jg. 59, Nr. 2, S. 15I-I89

McCloskey, Donald N. (1985): The Rhetoric of Economics, University of Wisconsin Press: Madison 
Mirowski, Philip (2002): Machine Dreams: Economics Becomes a Cyborg Science, New York: Cambridge University Press

Polanyi, Karl (1957): The Economy as Instituted Process, in: Granovetter, Mark/Swedberg, Richard (Hg.), The Sociology of Economic Life, Boulder: Westview Press, S. 29-5I

Reckling, Falk (2002): Interpretative Handlungsrationalität: Intersubjektivität als ökonomisches Problem und die Ressourcen der Hermeneutik, Marburg

Sen, Amartya Kumar (2000): Development as Freedom, New York: Anchor Books

Siler, Kyle (2003): The Social and Intellectual Organization and Construction of Economics, in: Post-Autistic Economics Review, Nr. 22, 24. November, Artikel 3

Smelser, Neil J./Swedberg, Richard (1994): The Handbook of Economic Sociology, Princeton: Princeton University Press

Solow, Robert (200I): L'économie entre empirisme et mathématisation, in: Le Monde, 2. Januar

Stiglitz, Joseph (1993): Post Walrasian and Post Marxian economics, in: Journal of Economic Perspectives, Jg. 7, Nr. I, S. I09-II4

The Cambridge 27 (200I): Opening Up Economics: A Proposal by Cambridge Students, in: Post-Autistic Economics Newsletter, Nr. 7, Io. Juli, Artikel 2

Woo, Henry K. H. (1986): What's Wrong with Formalization in Economics? An Epistemological Critique, Newark: Victoria Press

\section{Information und Kontakt}

Deutschsprachiger Raum:

Arbeitskreis Post-Autistische Ökonomie

Homepage: http://www.paecon.de

E-Mail: paecon@web.de

Weltweit:

Post-Autistic Economics

Homepage: http://www.paecon.net

E-Mail: edward.fullbrook@btinternet.com 